\title{
SOURCE-SUPERPOSITION METHOD OF SOLUTION OF A PERIODICALLY OSCILLATING WING AT SUPERSONIC SPEEDS*
}

BY

\section{H. J. STEWART AND TING-YI LI \\ California Institute of Technology}

Introduction and summary. In a recent paper Evvard (Ref. 1) discussed the linearized theory of the non-steady motion of three dimensional wings by methods which he had previously developed for the treatment of the corresponding steady flow problems (Refs. 2 and 3). Evvard represented the wing by a distribution of sources, and the important result of his steady state theory concerned the determination of the flow in a region influenced by a subsonic leading edge or wing tip. He showed that the influence of the flow around this subsonic edge of a flat lifting wing on the velocity potential at

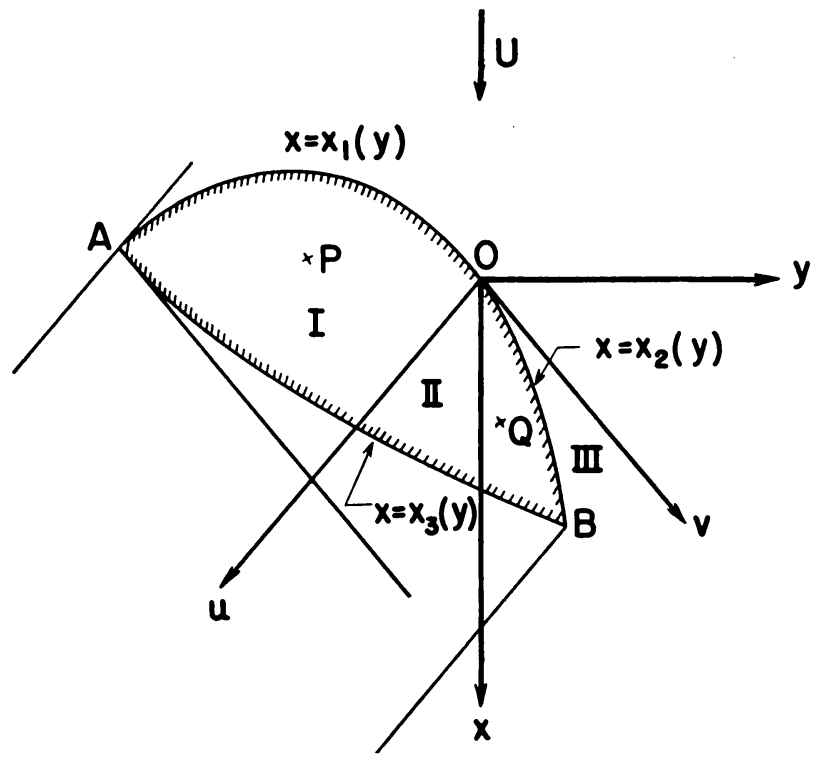

Fig. 1. Geometry of Wing.

a point within the region of influence of this edge is exactly equal to and of opposite sign to the contribution to the potential from the sources distributed over a simply determined region of the wing. In his paper on non-steady motion, he was able by similar methods to determine an explicit formula for the velocity potential; however he could not express the results in a similar, "equivalent area", form.

The present paper is concerned with the same problem of the non-steady lift of finite wings at supersonic speeds, particularly in regions which are influenced by subsonic leading edges or wing tips. It is shown that the simple "equivalent area" theorem developed by Evvard for the steady state case is also valid for oscillating wings. The

*Received December 30, 1949. 
theorem is not extended to arbitrary non-steady motions, and an example where the theorem in this simple form is apparently not valid is demonstrated.

The basic differential equation and boundary conditions. Consider a wing in a steady supersonic flow of velocity $U$ and Mach number $M$ in the direction of the x-axis. Then the velocity potential $\varphi$ which governs any small, possibly non-steady, disturbance produced by the wing satisfies the linearized differential equation

$$
\frac{1}{a^{2}} \frac{\partial^{2} \varphi}{\partial t^{2}}+\frac{2 U}{a^{2}} \frac{\partial^{2} \varphi}{\partial x \partial t}+\left(\frac{U^{2}}{a^{2}}-1\right) \frac{\partial^{2} \varphi}{\partial x^{2}}=\frac{\partial^{2} \varphi}{\partial y^{2}}+\frac{\partial^{2} \varphi}{\partial z^{2}},
$$

where $(x, y, z)$ are cartesian coordinates, $t$ is the time variable and $a$ is the speed of sound in the undisturbed flow so that $U=M a$. The wing is assumed to be near the plane $z=0$.

With the approximation of the linear equation, it is permissible to replace the boundary conditions at the point $(x, y, z)$ on the actual wing surface by the same boundary conditions applied on the plane $z=0$ at the point $(x, y, 0)$. In order to express the boundary conditions it is necessary in general to divide the wing surface into two different types of regions (see Fig. 1). The origin of the coordinate system is taken at the point 0 where the Mach line $0 v$ is tangent to the leading edge. The leading edge is thus divided into two segments, the segment $0 A$ which is defined by $x=x_{1}(y)$ or $y=y_{1}(x)$ and is a supersonic leading edge and the segment $0 B$ which is defined by $x=x_{2}(y)$ or $y=y_{2}(x)$ and is a subsonic leading edge. As a matter of convenience it is assumed that the trailing edge, $x=x_{3}(y)$, is a supersonic trailing edge where the Kutta condition need not apply. The Mach line $0 u$ then divides the wing into two regions. Region I, which is bounded by $x=x_{1}(y), x=x_{3}(y)$ and $0 u$, may be referred to as a purely supersonic region. Region II, which is bounded by $x=x_{2}(y), x=x_{3}(y)$ and $0 u$ may be referred to as a mixed supersonic region (Ref. 4).

At any point on the surface of the wing the flow must be tangential to the surface at any instant. This boundary condition, linearized, and applied to an oscillating condition is

$$
\begin{aligned}
& \frac{\partial \varphi_{T}}{\partial z}=w_{T}(x, y,+0) \exp (i \nu t)=U \Lambda_{T}(x, y,+0) \exp (i \nu t) \\
& \frac{\partial \varphi_{B}}{\partial z}=w_{B}(x, y,-0) \exp (i \nu t)=-U \Lambda_{B}(x, y,-0) \exp (i \nu t),
\end{aligned}
$$

where, except for the time factor, $w(x, y, z)$ is the $z$ component of the velocity and $\Lambda$ is the effective slope of the streamline and $\nu$ is the frequency of oscillation. The subscript $T$ refers to the top of the wing and the subscript $B$ refers to the bottom of the wing. In general $w_{T}$ and $w_{B}$ (or $\Lambda_{T}$ and $\Lambda_{B}$ ) need not be related. A sign convention for $\Lambda_{T}$ and $\Lambda_{B}$, adopted in Ref. 1 is also used here and is shown in Fig. 2.

From the definition of a purely supersonic region, there can be no disturbance in the flow ahead of the line $x=x_{1}(y)$. For any point $P$ in Region I the velocity $w$ is thus known at every point on the plane $z=0$ in the forward Mach cone from the point $P$. On the wing $w$ is given by Eq. 2 or Eq. 2a, ahead of the wing $w=0$.

For a point $Q$ in Region II conditions are more complex. As before, the velocity $w$ is given for that portion of the plane $z=0$ in the forward Mach cone from $Q$ which is 
covered by the wing by Eq. 2 or 2a. Also $w=0$ and $\varphi=0$ ahead of the line segments $x=x_{1}(y)$ and $0 v$. Since $x=x_{2}(y)$ is a subsonic leading edge, there is, in general, an interaction between the upper and lower surfaces which produces an upwash across the plane $z=0$ in Region III which is bounded by $x=x_{2}(y)$ and $0 v$. This upwash cannot, in general, be specified in advance. For this region the pressure must be continuous across the plane $z=0$ so the linearized boundary condition for this region is thus

$$
\frac{\partial \varphi_{T}}{\partial t}+U \frac{\partial \varphi_{T}}{\partial x}=\frac{\partial \varphi_{B}}{\partial t}+U \frac{\partial \varphi_{B}}{\partial x} .
$$

The boundary conditions on the plane $z=0$ for a point $Q$ in Region II are thus of a mixed type, involving $w$ over the wing, pressure over Region III and no disturbance ahead of the lines $0 v$ and $x=x_{1}(y)$.

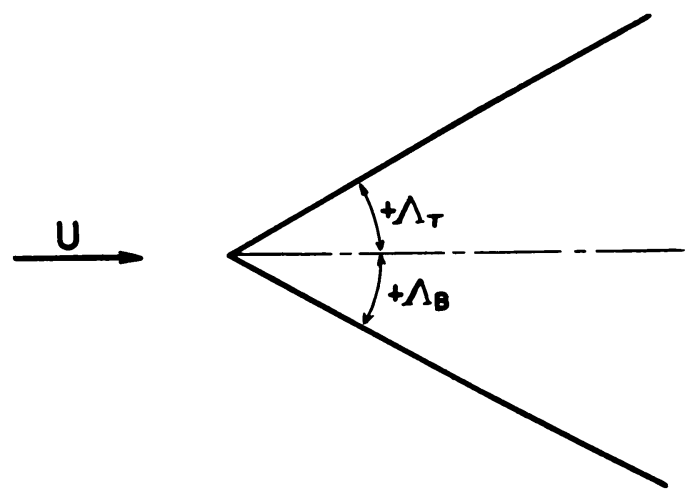

Fig. 2. Sign convention of $\Lambda$ 's.

Elementary oscillating source potential. Elementary solutions of Eq. 1 which can be superimposed to obtain more complex solutions can easily be obtained by the method of separation of variables. For this purpose it is convenient to introduce the following coordinate transformation:

$$
\begin{aligned}
& r=\left[x^{2}-\beta^{2}\left(y^{2}+z^{2}\right)\right]^{1 / 2}, \\
& \mu=\left[1-\frac{\beta^{2}\left(y^{2}+z^{2}\right)}{x^{2}}\right]^{-1 / 2}, \\
& \omega=\tan ^{-1}(z / y), \quad \tau=\beta^{2} a\left(t-\frac{M x}{\beta^{2} a}\right),
\end{aligned}
$$

where

$$
\beta^{2}=M^{2}-1 .
$$

These space variables were found useful in the treatment of steady conical flows (Ref. 5). The time transformation is similar to a combined Lorentz and Galilean transformation and has been used by Miles (Ref. 6). In these coordinates Eq. 1 is

$$
r^{2} \frac{\partial^{2} \varphi}{\partial \tau^{2}}=r^{2} \frac{\partial^{2} \varphi}{\partial r^{2}}+2 r \frac{\partial \varphi}{\partial r}+\frac{\partial}{\partial \mu}\left[\left(1-\mu^{2}\right) \frac{\partial \varphi}{\partial \mu}\right]+\frac{1}{1-\mu^{2}} \frac{\partial^{2} \varphi}{\partial \omega^{2}} .
$$

This is identical with the form of the classical wave equation in spherical coordinates. 
The solutions of Eq. 5, obtained by the method of separation of variables are

$$
\left.\varphi=\sum_{l, m, n} A_{l m n}\left\{\begin{array}{l}
\cos m \omega \\
\sin m \omega
\end{array}\right\}\left\{\begin{array}{l}
P_{n}^{m}(\mu) \\
Q_{n}^{m}(\mu)
\end{array}\right\} \begin{array}{l}
r^{-1 / 2} J_{-n-1 / 2}(l r) \\
r^{-1 / 2} J_{n+1 / 2}(l r)
\end{array}\right\} \exp ( \pm i l \tau),
$$

where $l, m$ and $n$ are the separation parameters. $P_{n}^{m}(\mu)$ and $Q_{n}^{m}(\mu)$ are Associated Legendre functions and $J_{\star(n+1 / 2)}(l r)$ is a Bessel function of order $\pm(n+1 / 2)$.

For $m=n=0$, a simple solution of Eq. 6 is

$$
\varphi_{1}=A r^{-1 / 2} J_{-1 / 2}(l r) \exp (i l \tau) .
$$

If $\tau$ is replaced by the physical time variable from Eq. 4, the Bessel function is written in the trigonometric form and $l$ is eliminated by the relation $\nu=l \beta^{2} a$, Eq. 7 becomes

$$
\varphi_{1}=\frac{A_{1}}{r} \cos \left(\frac{\nu r}{\beta^{2} a}\right) \exp \left[i \nu\left(t-\frac{U x}{\beta^{2} a^{2}}\right)\right],
$$

where $A_{1}$ is a new arbitrary constant. Equation 8 may be considered as defining a supersonic oscillating source. This basic solution has been used in this form by Miles (Ref. 7). If the basic solutions used by Garrick and Rubinow (Ref. 4) or by Evvard (Ref. 1) are applied to oscillating problems, they can be reduced to this same form. It may be noted that for $\nu=0 \mathrm{Eq} .8$ reduces to the usual steady state source potential. The complete velocity potential field for an oscillating source is defined by Eq. 8 in the downstream Mach cone and as zero outside the Mach cone.

Velocity potential of an oscillating wing. For a point in the purely supersonic region the velocity potential due to the wing can readily be obtained by replacing the wing

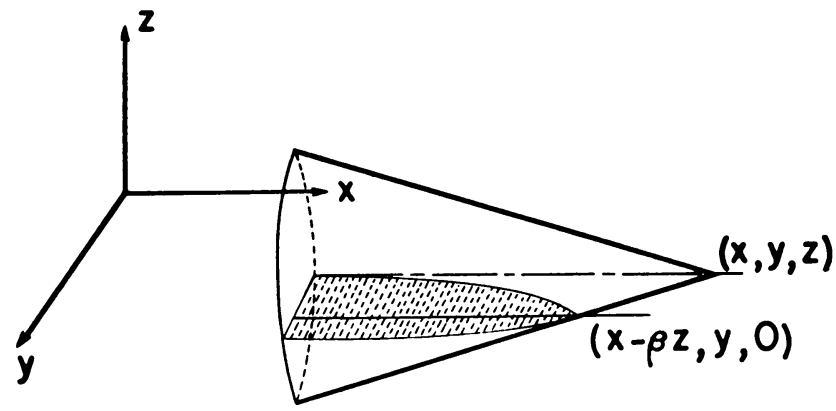

FIG. 3. Singularities or sources in the $x, y$ plane, that affect conditions at $(x, y, z)$ at instant $t$.

by a distribution of sources over the wing surface. If the region of dependence of a given point includes only that portion of the wing which is purely supersonic, the velocity potential for $z \geq 0$ due to the source distribution is thus

$$
\Phi(x, y, z, t)=\iint_{s} A_{T}(\xi, \eta) \exp \left\{i \nu\left[t-\frac{U(x-\xi)}{\beta^{2} a^{2}}\right]\right\} \cos \left(\frac{\nu r_{1}}{\beta^{2} a}\right) \frac{d \xi d \eta}{r_{1}}
$$

where

$$
r_{1}=\left[(x-\xi)^{2}-\beta^{2}(y-\eta)^{2}-\beta^{2} z^{2}\right]^{1 / 2} .
$$

Here $A_{T}(\xi, \eta)$ is the source strength per unit area at the coordinate $(\xi, \eta)$ on the wing surface. The region of dependence, which determines the region of integration on the wing surface, is bounded on the downstream side by the line $r_{1}=0$ (Fig. 3). 
If the source strength $A_{T}(\xi, \eta)$ can be chosen so the boundary conditions for the purely supersonic region are satisfied, Eq. 9 is the proper solution. In order to do this it is convenient to replace the integration variable $\eta$ by

$$
\beta(y-\eta)=-r_{2} \sin \theta
$$

where

$$
r_{2}=\left[(x-\xi)^{2}-\beta^{2} z^{2}\right]^{1 / 2} .
$$

With this notation Eq. 9 becomes

$$
\begin{aligned}
\Phi(x, y, z, t)=\frac{1}{\beta} \exp (i \nu t) \int_{\xi_{1}}^{x-\beta_{z}} \exp \left[-\frac{i \nu U}{\beta^{2} a^{2}}(x-\xi)\right] d \xi \\
\times \int_{-\pi / 2}^{\pi / 2} A_{T}\left(\xi, y+\frac{r_{2}}{\beta} \sin \theta\right) \cos \left(\frac{\nu r_{2}}{\beta^{2} a} \cos \theta\right) d \theta,
\end{aligned}
$$

where $\xi_{1}$ is the least value of $\xi$ on the leading edge. Since

$$
\begin{gathered}
\frac{\partial r_{2}}{\partial z}=-\frac{\beta^{2} z}{r_{2}} \\
\frac{\partial \Phi}{\partial z}=-\pi A_{T}(x-\beta z, y) \exp (i \nu t) \\
-\beta z \exp (i \nu t) \int_{\xi_{1}}^{x-\beta z} \exp \left[-\frac{i \nu U}{\beta^{2} a^{2}}(x-\xi)\right] \frac{1}{r_{2}} d \xi \\
\times \int_{-\pi / 2}^{\pi / 2} \frac{\partial}{\partial r_{2}}\left[A_{T}\left(\xi, y+\frac{r_{2}}{\beta} \sin \theta\right) \cos \left(\frac{\nu r_{2}}{\beta^{2} a} \cos \theta\right)\right] d \theta .
\end{gathered}
$$

If the function $A_{T}(\xi, \eta)$ is continuous in the neighborhood of the point $(x, y)$, the magnitude of the double integral in Eq. 15 is finite for sufficiently small values of $z$; so

$$
\lim _{z \rightarrow+0}\left(\frac{\partial \Phi}{\partial z}\right)=-\pi A_{T}(x, y) \exp (i \nu t) .
$$

By comparison of Eqs. 16 and 2, it is seen that

$$
A_{T}(x, y)=-\frac{1}{\pi} w_{T}(x, y,+0)=-\frac{U}{\pi} \Lambda_{T}(x, y,+0) .
$$

For a point below the wing, $z \leq 0$, a similar analysis shows that

$$
A_{B}(x, y)=\frac{1}{\pi} w_{B}(x, y,-0)=-\frac{U}{\pi} \Lambda_{B}(x, y,-0)
$$

The required source strength, $A(x, y)$ in the plane $z=0$ is thus completely determined for any point in the purely supersonic region. On the wing $A(x, y)$ is given by Eq. 17 or Eq. 17a. Ahead of the wing the disturbance $(\partial \Phi / \partial z)_{z=0}$ is zero; so $A(x, y)=0$ in this region. With these values of $A(x, y)$, Eq. 9 defines the velocity potential and thus the velocity components and the pressure on the wing. This analysis was given in a similar form by Miles in Ref. 6 (some errors in his presentation were corrected in Ref. 7). 
The results of this analysis may be summarized in the following two theorems:

Theorem 1. The strength of the source at any point at any instant on the surface of an oscillating wing is linearly dependent on the downwash at that point and at that instant and is independent of the downwash of the neighboring points.

Theorem 2. The velocity potential at instant $t$, at a point $P$ in the purely supersonic region of the surface of a three-dimensional oscillating wing (Fig. 1) may be computed by

$$
\begin{aligned}
& \Phi(x, y, \pm 0, t)=-\frac{U}{\pi} \exp (i \nu t) \int_{\xi_{1}}^{x} \exp \left[-i \nu \frac{U}{\beta^{2} a^{2}}(x-\xi)\right] d \xi \\
& \quad \times \int_{\nu-(x-\xi) / \beta}^{\nu+(x-\xi) / \beta}\left\{\begin{array}{l}
\Lambda_{T}(\xi, \eta) \\
\Lambda_{B}(\xi, \eta)
\end{array}\right\} \frac{\cos \left\{\left(\nu / \beta^{2} a\right)\left[(x-\xi)^{2}-\beta^{2}(y-\eta)^{2}\right]^{1 / 2}\right\}}{\left[(x-\xi)^{2}-\beta^{2}(y-\eta)^{2}\right]^{1 / 2}} d \eta,
\end{aligned}
$$

where $z= \pm 0$ refers to the $\left\{\begin{array}{l}\text { top } \\ \text { bottom }\end{array}\right\}$ surface of the wing.

A mixed supersonic region may be converted into a "psuedo-purely supersonic region" by Evvard's procedure of inserting a diaphragm into Region III of Fig. 1 which is an extension of the wing having the following properties:

a) It does not change the flow over the wing

b) It sustains no lift

With this supposition, the top and bottom surfaces of the wing may again be considered to be independent so that Eq. 9a applies; however the diaphragm slope is in general an unknown function which must be determined.

A part of the wing in Fig. 1 is shown enlarged in Fig. 4. In order to compute the

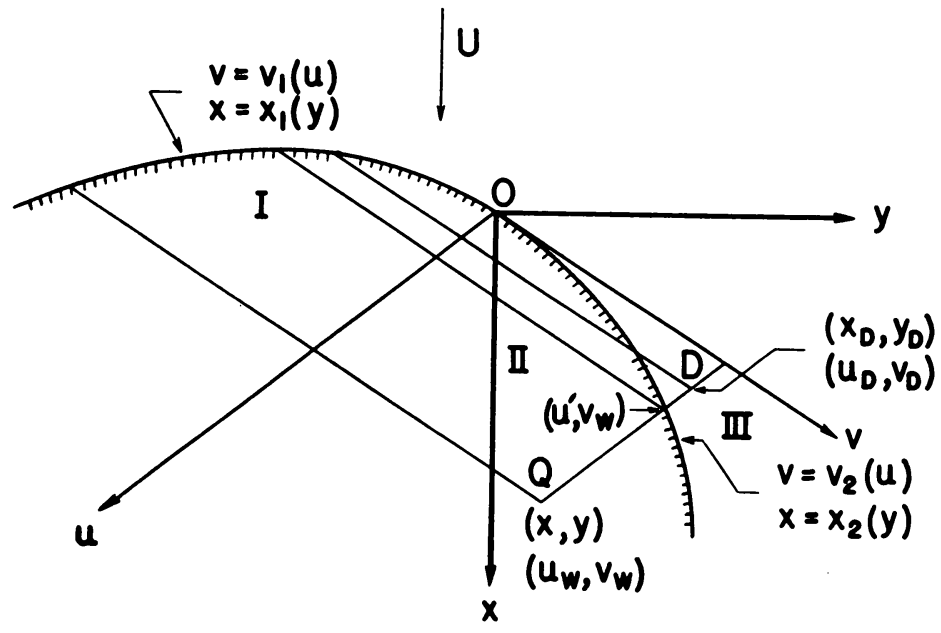

FIG. 4. A portion of the wing in Figure 1

velocity potential at the point $Q$, it is convenient to first consider a point $D$ located on the trace of the upstream facing Mach cone from $Q$, in the diaphragm plane. Let the unknown downwash and the effective slope of the streamline on the top surface of the 
diaphragm be $w_{D T}\left(x_{D}, y_{D}\right)$ and $\Lambda_{D r}\left(x_{D}, y_{D}\right)$ respectively. Then, by Eqs. 9 and 17, it is found that

$$
\begin{aligned}
& \Phi_{D}\left(x_{D}, y_{D},+0, t\right)=-\frac{1}{\pi} \exp (i \nu t) \\
& \quad \times \iint_{S_{W}} w_{T}(\xi, \eta) \frac{\cos \left\{\left(\nu / \beta^{2} a\right)\left[\left(x_{D}-\xi\right)^{2}-\beta^{2}\left(y_{D}-\eta\right)^{2}\right]^{1 / 2}\right.}{\left[\left(x_{D}-\xi\right)^{2}-\beta^{2}\left(y_{D}-\eta\right)^{2}\right]^{1 / 2} \exp \left[i \nu\left(U / \beta^{2} a^{2}\right)\left(x_{D}-\xi\right)\right]} d \xi d \eta \\
& \quad-\frac{1}{\pi} \exp (i \nu t) \\
& \quad \times \iint_{S_{D}} w_{D T}(\xi, \eta) \frac{\cos \left\{\left(\nu / \beta^{2} a\right)\left[\left(x_{D}-\xi\right)^{2}-\beta^{2}\left(y_{D}-\eta\right)^{2}\right]^{1 / 2}\right\}}{\left[\left(x_{D}-\xi\right)^{2}-\beta^{2}\left(y_{D}-\eta\right)^{2}\right]^{1 / 2}} \frac{\exp \left[i \nu\left(U / \beta^{2} a^{2}\right)\left(x_{D}-\xi\right)\right]}{x^{2}} d \xi d \eta,
\end{aligned}
$$

where $S_{W}$ is the region of the wing and $S_{D}$ is the region of the diaphragm included in the upstream facing Mach cone from $D\left(x_{D}, y_{D},+0\right)$, at instant $t$. The regions of integration $S_{W}$ and $S_{D}$ are most easily expressed in terms of the oblique $u, v$ coordinates defined as follows:

$$
\begin{aligned}
& u=\frac{M}{2 \beta}(\xi-\beta \eta), \quad \xi=\frac{\beta}{M}(v+u), \\
& \text { or } \\
& v=\frac{M}{2 \beta}(\xi+\beta \eta), \quad \eta=\frac{1}{M}(v-u),
\end{aligned}
$$

With these coordinate transformations, the point $\left(x_{D}, y_{D}\right)$ is transformed into $\left(u_{D}, v_{D}\right)$, where

$$
\begin{array}{lll}
u_{D}=\frac{M}{2 \beta}\left(x_{D}-\beta y_{D}\right) & & x_{D}=\frac{\beta}{M}\left(v_{D}+u_{D}\right) \\
v_{D}=\frac{M}{2 \beta}\left(x_{D}+\beta y_{D}\right) & & y_{D}=\frac{1}{M}\left(v_{D}-u_{D}\right)
\end{array}
$$

The surface integral $\Phi_{D_{r}}$ in Eq. 18 will now be integrated in the $u, v$ plane. Then, Eq. 18 becomes

$$
\begin{aligned}
& \Phi_{D_{T}}\left(u_{D}, v_{D},+0, t\right)=-\frac{1}{\pi M} \exp (i \nu t) \int_{0}^{u D} \frac{d u}{\left(u_{D}-u\right)^{1 / 2}} \frac{d u}{\exp \left[(i v / \beta a)\left(u_{D}-u\right)\right]} \\
& \quad \times \int_{v_{1}(u)}^{v_{\mathcal{B}}(u)} \frac{W_{T}(u, v) \cos \left\{(2 \nu / M \beta a)\left[\left(u_{D}-u\right)\left(v_{D}-v\right)\right]^{1 / 2}\right\}}{\left(v_{D}-v\right)^{1 / 2} \exp \left[(i v / \beta a)\left(v_{D}-v\right)\right]} d v \\
& \quad-\frac{1}{\pi M} \exp (i \nu t) \int_{0}^{u_{D}} \frac{d u}{\left(u_{D}-u\right)^{1 / 2} \exp \left[(i v / \beta a)\left(u_{D}-u\right)\right]} \\
& \quad \times \int_{v_{\mathbf{s}}(u)}^{v_{D}} \frac{W_{D_{T}}(u, v) \cos \left\{(2 \nu / M \beta a)\left[\left(u_{D}-u\right)\left(v_{D}-v\right)\right]^{1 / 2}\right\}}{\left(v_{D}-v\right)^{1 / 2} \exp \left[(i v / \beta a)\left(v_{D}-v\right)\right]} d v .
\end{aligned}
$$

where $w_{T}(u, v)$ is the downwash on the top surface of the wing, $w_{D r}(u, v)$ is the down- 
wash on the top surface of the diaphragm, the area bounded by $0 \leq u \leq u_{D}$ and $v_{1}(u) \leq$ $v \leq v_{2}(u)$ is $S_{W}$, and the area bounded by $0 \leq u \leq u_{D}$ and $v_{2}(u) \leq v \leq v_{D}$ is $S_{D}$ (Fig. 4).

Similarly, for the corresponding point $D\left(u_{D}, v_{D},-0, t\right)$ on the bottom surface of the diaphragm, it is seen that

$$
\begin{aligned}
& \Phi_{D_{B}}\left(u_{D}, v_{D},-0, t\right)=\frac{1}{\pi M} \exp (i \nu t) \int_{0}^{u_{D}} \overline{\left(u_{D}-\bar{u}\right)^{1 / 2}} \frac{d u}{\exp \left[(i \nu / \beta a)\left(u_{D}-u\right)\right]}
\end{aligned}
$$

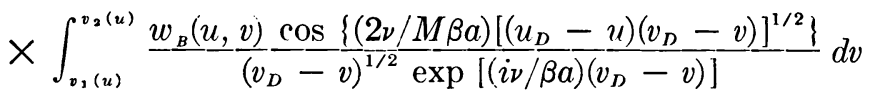

$$
\begin{aligned}
& +\frac{1}{\pi M} \exp (i \nu t) \int_{0}^{u_{D}} \frac{d u}{\left(u_{D}-u\right)^{1 / 2}} \frac{d u}{\exp \left[(i \nu / \beta a)\left(u_{D}-u\right)\right]} \\
& \times \int_{v_{\mathbf{z}}(u)}^{v_{D}} \frac{w_{D_{B}}(u, v) \cos \left\{(2 \nu / M \beta a)\left[\left(u_{D}-u\right)\left(v_{D}-v\right)\right]^{1 / 2}\right\}}{\left(v_{D}-v\right)^{1 / 2} \exp \left[(i \nu / \beta a)\left(v_{D}-v\right)\right]} d v .
\end{aligned}
$$

Off the wing, the downwash must be continuous. In terms of the effective slopes of the stream lines, this condition is, with the sign convention of Fig. 2,

$$
\Lambda_{D T}(u, v)=-\Lambda_{D_{B}}(u, v)=\Lambda_{D}(u, v) \text {. }
$$

From Eq. 3 it is found that in Region III

$$
\Phi_{D_{T}}(x, y,+0, t)=\Phi_{D_{B}}(x, y,-0, t)+F(x-U t, y),
$$

where $F$ is an integration function. The foremost Mach cone (Fig. 4) from the origin, 0 , represents a line of infinitesimal disturbance along which $F(x-U t, y)$ can be set equal to zero at all times. $F$ remains zero along $y=$ constant lines for values of $x$ not intercepted by the wing (Ref. 1). Therefore, in Eq. 24, $F$ may be put to zero. Behind a trailing edge, $F$ may be different from zero and the theory must be modified. Then, from Eqs. 21, 22, 23 and 24 (with $F=0$ ), it is seen that

$$
\begin{aligned}
\frac{1}{2} \int_{0}^{u_{D}} & \frac{d u}{\left(u_{D}-u\right)^{1 / 2} \exp \left[(i \nu / \beta a)\left(u_{D}-u\right)\right]} \\
& \times \int_{v_{1}(u)}^{v_{2}(u)} \frac{\left[\Lambda_{B}(u, v)-\Lambda_{T}(u, v)\right] \cos \left\{(2 \nu / M \beta a)\left[\left(u_{D}-u\right)\left(v_{D}-v\right)\right]^{1 / 2}\right\}}{\left(v_{D}-v\right)^{1 / 2} \exp \left[(i \nu / \beta a)\left(v_{D}-v\right)\right]} d v \\
\quad & \int_{0}^{u_{D}} \frac{d u}{\left(u_{D}-u\right)^{1 / 2} \exp \left[(i \nu / \beta a)\left(u_{D}-u\right)\right]} \\
& \times \int_{v_{2}(u)}^{v_{D}} \frac{\Lambda_{D}(u, v) \cos \left\{(2 \nu / M \beta a)\left[\left(u_{D}-u\right)\left(v_{D}-v\right)\right]^{1 / 2}\right\}}{\left(v_{D}-v\right)^{1 / 2} \exp \left[(i \nu / \beta a)\left(v_{D}-v\right)\right]} d v .
\end{aligned}
$$

When $\nu=0$, this reduces to

$$
\begin{gathered}
\frac{1}{2} \int_{0}^{u_{D}} \frac{d u}{\left(u_{D}-u\right)^{1 / 2}} \int_{v_{1}(u)}^{v_{2}(u)} \frac{\Lambda_{B}(u, v)-\Lambda_{T}(u, v)}{\left(v_{D}-v\right)^{1 / 2}} d v \\
=\int_{0}^{u_{D}} \frac{d u}{\left(u_{D}-u\right)^{1 / 2}} \int_{v_{2}(u)}^{v_{D}} \frac{\Lambda_{D}(u, v)}{\left(v_{D}-v\right)^{1 / 2}} d v .
\end{gathered}
$$


Inasmuch as the limits of integration of the $u$-integrals are the same for all values of $u_{D}$ and the integrals are "faltung" integrals, the two integrals with respect to $v$ may be equated along lines of constant $u$ that extends across the wing and the diaphragm (Fig. 4). Therefore,

$$
\int_{v_{\mathbf{2}}(u)}^{v_{D}} \frac{\Lambda_{D}(u, v)}{\left(v_{D}-v\right)^{1 / 2}} d v=\int_{v_{1}(u)}^{v_{\text {( }}(u)} \frac{\Lambda_{B}(u, v)-\Lambda_{T}(u, v)}{2\left(v_{D}-v\right)^{1 / 2}} d v .
$$

This is the fundamental result of Ref. 2 and also is the basic equation of Ref. 3. The above argument is valid because the terms containing $u_{D}$ do not appear in the $v$-integrals, and hence Eq. 27 is true for all $u_{D}$ 's on the line $v=v_{D}$.

The parallel treatment of Eq. 25 would be possible if the terms containing $\left(u_{D}-u\right)$ $\left(v_{D}-v\right)$ can be separated as in Eq. 26, under the integral signs. The present treatment represents a first attempt towards this end. The isolation of terms containing $\left(u_{D}-u\right)$ from terms containing $\left(v_{D}-v\right)$ such that the $v$-integral is free of the $\left(u_{D}-u\right)$ factor, may be accomplished by the following procedures.

The term $\left\{\left(v_{D}-v\right)\left(u_{D}-u\right)\right\}^{1 / 2}$ vanishes at $\left(u_{D}, v_{D}\right)$, therefore Eq. 25 actually should be

$$
\begin{aligned}
\lim _{\epsilon \rightarrow 0} & \int_{0}^{u_{D}-\epsilon} \frac{d u}{\left(u_{D}-u\right)^{1 / 2} \exp \left[(i v / \beta a)\left(u_{D}-u\right)\right]} \\
& \times \int_{v_{1}(u)}^{v_{*}(u)} \frac{\left[\Lambda_{B}(u, v)-\Lambda_{T}(u, v)\right] \cos \left\{(2 v / M \beta a)\left[\left(u_{D}-u\right)\left(v_{D}-v\right)\right]^{1 / 2}\right\}}{2\left(v_{D}-v\right)^{1 / 2} \exp \left[(i v / \beta a)\left(v_{D}-v\right)\right]} d v \\
& =\lim _{\substack{\epsilon \rightarrow 0 \\
\epsilon_{1} \rightarrow 0}} \int_{0}^{u_{D}-\epsilon} \frac{d u}{\left(u_{D}-u\right)^{1 / 2} \exp \left[(i v / \beta a)\left(u_{D}-u\right)\right]} \\
& \times \int_{v_{2}(u)}^{v_{D-\epsilon}} \frac{\Lambda_{D}(u, v) \cos \left\{(2 \nu / M \beta a)\left[\left(u_{D}-u\right)\left(v_{D}-v\right)\right]^{1 / 2}\right\}}{\left(v_{D}-v\right)^{1 / 2} \exp \left[(i v / \beta a)\left(v_{D}-v\right)\right]} d v .
\end{aligned}
$$

The nature of the functions $\Lambda_{B}, \Lambda_{T}$ and $\Lambda_{D}$ must be such as to insure the existence of the improper integrals. Thus, except for the singularity $\left(u_{D}, v_{D}\right)$; in the finite integration regions, the integrands are defined and bounded everywhere. Now, the circular functions are defined by power series; in particular, the power series expansion of the cosine function is

$$
\cos z=\sum_{n=0}^{\infty} \frac{(-)^{n}}{(2 n) !} z^{2 n}
$$

The series (28) has the following properties (Ref. 8):

(1) It converges absolutely for all values of $z$ (real and complex),

(2) It converges uniformly in any bounded domain of values of $z$, and consequently,

(3) It is a continuous function of $z$ for all values of $z$.

Because of the uniform continuity, the cosine function in Eq. 25a may be expanded in an infinite series and the orders of integration and summation may be inverted. Thus 


$$
\begin{aligned}
\sum_{n=0}^{\infty} & \frac{(-)^{n}(\nu / \beta a)^{2 n}(1 / M)^{2 n} \pi^{1 / 2}}{n ! \Gamma(n+1 / 2)} \\
& \times \lim _{\epsilon \rightarrow 0} \int_{0}^{u_{D}-\epsilon} \frac{\left(u_{D}-u\right)^{n-1 / 2} d u}{\exp \left[(i \nu / \beta a)\left(u_{D}-u\right)\right]} \int_{v_{,}(u)}^{\nu_{3}(u)} \frac{\left[\Lambda_{B}(u, v)-\Lambda_{T}(u, v)\right]\left(v_{D}-v\right)^{n-1 / 2}}{2 \exp \left[(i \nu / \beta a)\left(v_{D}-v\right)\right]} d v \\
& =\sum_{n=0}^{\infty} \frac{(-)^{n}(\nu / \beta a)^{2 n}(1 / M)^{2 n} \pi^{1 / 2}}{n ! \Gamma(n+1 / 2)} \\
& \times \lim _{\epsilon \rightarrow 0} \int_{0}^{u_{D}-\epsilon} \frac{\left(u_{D}-u\right)^{n-1 / 2} d u}{\exp \left[(i \nu / \beta a)\left(u_{D}-u\right)\right]} \lim _{\epsilon_{1} \rightarrow 0} \int_{v_{2}(u)}^{v_{D}-\epsilon_{1}} \frac{\Lambda_{D}(u, v)\left(v_{D}-v\right)^{n-1 / 2}}{\exp \left[(i \nu / \beta a)\left(v_{D}-v\right)\right]} d v .
\end{aligned}
$$

With the conviction that the improper integrals under question exist, the "lim" signs may be left out.

In Eq. 25b, unlike in Eq. 25a, the $v$-integrals do not contain $u_{D}$ terms, and the problem has been reduced to one analogous to that of Eq. 26. Now, it may be pointed out that since Eq. 25a is derived by equating the velocity potential on the top and bottom surfaces of the diaphragm in Region III (Fig. 4), the two sides of Eq. 25b may be conveniently considered as power series in $(1 / M)$ of a potential function $\Phi$, satisfying the original linear differential equation, Eq. 1; consequently corresponding terms may be equated.

Therefore, for constant value of $v_{D}$, with $n$ being any positive integer,

$$
\int_{v_{\mathbf{s}(u)}}^{v_{D}} \frac{\Lambda_{D}(u, v)\left(v_{D}-v\right)^{n-1 / 2}}{\exp \left[(i \nu / \beta a)\left(v_{D}-v\right)\right]} d v=\int_{v_{1}(u)}^{v_{\mathbf{s}}(u)} \frac{\left[\Lambda_{B}(u, v)-\Lambda_{T}(u, v)\right]\left(v_{D}-v\right)^{n-1 / 2}}{2 \exp \left[(i \nu / \beta a)\left(v_{D}-v\right)\right]} d v .
$$

In this system of simultaneous integral equations $\Lambda_{B}(u, v)$ and $\Lambda_{T}(u, v)$ are known while $\Lambda_{D}(u, v)$ is unknown. Consider, say, $(N+1)$ integral equations corresponding to $n=0,1,2, \cdots N$. (Of course, in the limit, $N \rightarrow \infty$ ). In order that these $(N+1)$ simultaneous equations may determine one unknown $\Lambda_{D}$, it is necessary that the $(N+1)$ equations are not mutually independent, that is, the $(N+1)$ equations are reducible to one equation. In fact, this is true for the given system. For instance, when $n=1$, it is obtained from Eq. 29 that

$$
\int_{v_{2}(u)}^{v_{D}} \frac{\Lambda_{D}(u, v)\left(v_{D}-v\right)^{1 / 2} d v}{\exp \left[(i \nu / \beta a)\left(v_{D}-v\right)\right]}=\int_{v_{1}(u)}^{v_{2}(u)} \frac{\left[\Lambda_{B}(u, v)-\Lambda_{T}(u, v)\right]\left(v_{D}-v\right)^{1 / 2} d v}{2 \exp \left[(i \nu / \beta a)\left(v_{D}-v\right)\right]} .
$$

Carry out a differentiation of Eq. 30 with respect to $v_{D}$. The result of this differentiation plus $(i \nu / \beta a)$ times Eq. 30 yields

$$
\int_{v_{1}(u)}^{v_{D}} \frac{\Lambda_{D}(u, v)\left(v_{D}-v\right)^{-1 / 2} d v}{\exp \left[(i \nu / \beta a)\left(v_{D}-v\right)\right]}=\int_{v_{1}(u)}^{v_{2}(u)} \frac{\left[\Lambda_{B}(u, v)-\Lambda_{T}(u, v)\right]\left(v_{D}-v\right)^{-1 / 2} d v}{2 \exp \left[(i \nu / \beta a)\left(v_{D}-v\right)\right]}
$$

which is Eq. 29 for $n=0$. Therefore, when $\Lambda_{D}$ satisfies Eq. 30, it also satisfies Eq. 31 . This argument can be carried on, by induction, to include the case for every $n$. Therefore, the system given by Eq. 29 is consistent and determines an unique function $\Lambda_{D}$.

For the determination of the contribution of the diaphragm on the velocity potential at a point $Q\left(u_{w}, v_{W}, \pm 0\right)$ on the top or bottom surface of the wing, it is not necessary 
to solve the integral equation, Eq. 29, explicitly. Let this contribution be called $\Phi_{W_{D}}\left(u_{W}, v_{W}, \pm 0, t\right)$ (see Fig. 4). Then,

$$
\begin{gathered}
\Phi_{W_{D}}\left(u_{W}, v_{W}, \pm 0, t\right)=\mp \frac{U}{\pi M} \exp (i v t) \int_{0}^{u^{\prime}} \frac{d u}{\left(u_{W}-u\right)^{1 / 2} \exp \left[(i v / \beta a)\left(u_{W}-u\right)\right]} \\
\times \int_{v_{2}(u)}^{\sigma_{W}} \frac{\Lambda_{D}(u, v) \cos \left\{(2 \nu / M \beta a)\left[\left(u_{W}-u\right)\left(v_{W}-v\right)\right]^{1 / 2}\right\} d v}{\left(v_{W}-v\right)^{1 / 2} \exp \left[(i v / \beta a)\left(v_{W}-v\right)\right]} \\
=\mp \frac{U}{\pi M} \exp (i v t) \sum_{n=0}^{\infty} \frac{(-)^{n}(\nu / \beta a)^{2 n}(1 / M)^{2 n} \pi^{1 / 2}}{n ! \Gamma(n+1 / 2)} \\
\times \int_{0}^{u^{\prime}} \frac{\left(u_{W}-u\right)^{n-1 / 2} d u}{\exp \left[(i v / \beta a)\left(u_{W}-u\right)\right]} \int_{v_{2}(u)}^{v_{W}} \frac{\Lambda_{D}(u, v)\left(v_{W}-v\right)^{n-1 / 2} d v}{\exp \left[(i v / \beta a)\left(v_{W}-v\right)\right]}
\end{gathered}
$$

where $u^{\prime}$ is the $u$-coordinate of the intersection point of the curves: $v=v_{2}(u)$ and $v=$ $v_{W}$, i.e. $v_{2}\left(u^{\prime}\right)=v_{W}$.

By comparing

$$
\int_{v_{2}(u)}^{v_{W}} \frac{\Lambda_{D}(u, v)\left(v_{W}-v\right)^{n-1 / 2}}{\exp \left[(i v / \beta a)\left(v_{W}-v\right)\right]} d v \quad \text { with } \quad \int_{v_{3}(u)}^{v_{D}} \frac{\Lambda_{D}(u, v)\left(v_{D}-v\right)^{n-1 / 2}}{\exp \left[(i v / \beta a)\left(v_{D}-v\right)\right]} d v,
$$

it is seen that they are identical if every $v_{D}$ in the latter is replaced by $v_{W}$. But the value of $v_{D}$ along the $v=$ constant line passing through the point $\left(u_{W}, v_{W}, \pm 0\right)$ is $v_{W}$ (Fig. 4). Hence $v_{D}$ may be replaced by $v_{W}$ in Eq. 29 and Eq. 32 becomes

$$
\begin{gathered}
\Phi_{W_{D}}\left(u_{W}, v_{W}, \pm 0, t\right)=\mp \frac{U}{\pi M} \exp (i \nu t) \sum_{n=0}^{\infty} \frac{(-)^{n}(\nu / \beta a)^{2 n}(1 / M)^{2 n} \pi^{1 / 2}}{n ! \Gamma(n+1 / 2)} \\
\times \int_{0}^{u^{\prime}} \frac{\left(u_{W}-u\right)^{n-1 / 2} d u}{\exp \left[(i \nu / \beta a)\left(u_{W}-u\right)\right]} \int_{v_{1}(u)}^{v_{2}(u)} \frac{\left[\Lambda_{B}(u, v)-\Lambda_{T}(u, v)\right]\left(v_{W}-v\right)^{n-1 / 2}}{2 \exp \left[(i v / \beta a)\left(v_{W}-v\right)\right]} d v \\
=\mp \frac{U}{\pi M} \exp (i \nu t) \int_{0}^{u^{\prime}} \frac{d u}{\left(u_{W}-u\right)^{1 / 2}} \operatorname{exp[(iv/\beta a)(u_{W}-u)]} \\
\times \int_{v_{1}(u)}^{v_{2}(u)} \frac{\left[\Lambda_{B}(u, v)-\Lambda_{T}(u, v)\right] \cos }{2\left(v_{W}-v\right)^{1 / 2}} \frac{\left\{(2 \nu / M \beta a)\left[\left(u_{W}-u\right)\left(v_{W}-v\right)\right]^{1 / 2}\right\}}{\exp \left[(i \nu / \beta a)\left(v_{W}-v\right)\right]} d v .
\end{gathered}
$$

In Eq. 33 an important theorem is established. The theorem may be stated as follows:

Theorem 3. In the computation of the velocity potential at an instant $t$ at a point $Q$ in the mixed supersonic region of an oscillating wing at supersonic speed, the contribution of the diaphragm may be evaluated by Eq. 33. In other words, the contribution of the diaphragm can be evaluated by an equivalent integration over a portion of the wing surface. Now, the velocity potential $\Phi$ at point $Q$ on the top wing surface at instant $t$ may be computed. It is (Fig. 4) 


$$
\begin{aligned}
& \Phi\left(u_{W}, v_{W},+0, t\right) \\
& =-\frac{U}{\pi M} \exp (i \nu t) \int_{0}^{u^{\prime}} \frac{d u}{\left(u_{W}-u\right)^{1 / 2} \exp \left[(i \nu / \beta a)\left(u_{W}-u\right)\right]} \\
& \times \int_{v_{1}(u)}^{v_{B}(u)} \frac{\left[\Lambda_{B}(u, v)-\Lambda_{T}(u, v)\right] \cos \left\{(2 \nu / M \beta a)\left[\left(u_{W}-u\right)\left(v_{W}-v\right)\right]^{1 / 2}\right\}}{2\left(v_{W}-v\right)^{1 / 2} \exp \left[(i \nu / \beta a)\left(v_{W}-v\right)\right]} d v \\
& -\frac{U}{\pi M} \exp (i \nu t) \int_{0}^{u^{\prime}} \frac{d u}{\left(u_{W}-u\right)^{1 / 2} \exp \left[(i \nu / \beta a)\left(u_{W}-u\right)\right]} \\
& \times \int_{v_{1}(u)}^{v_{8}(u)} \frac{\Lambda_{T}(u, v)}{\left(v_{W}-v\right)^{1 / 2} \cos \left\{(i \nu / \beta a)\left(v_{W}-v\right)\right]} d v \\
& -\frac{U}{\pi M} \exp (i \nu t) \int_{u^{\prime}}^{u_{W}} \frac{d u}{\left(u_{W}-u\right)^{1 / 2}} \frac{d u}{\exp \left[(i \nu / \beta a)\left(u_{W}-u\right)\right]} \\
& \times \int_{v_{1}(u)}^{v_{W}} \frac{\Lambda_{T}(u, v) \cos \left\{(2 \nu / M \beta a)\left[\left(u_{W}-u\right)\left(v_{W}-v\right)\right]^{1 / 2}\right\}}{\left(v_{W}-v\right)^{1 / 2} \exp \left[(i v / \beta a)\left(v_{W}-v\right)\right]} d v .
\end{aligned}
$$

In Eq. 34 the first surface integral represents the contribution from the diaphragm, while the last two surface integrals are the contribution from the top surface of the wing. By combining the first and second surface integrals, it is seen that

$$
\begin{aligned}
\Phi\left(u_{W}, v_{W},+0, t\right) \\
=-\frac{U}{\pi M} \exp (i \nu t) \int_{0}^{u^{\prime}} \frac{d u}{\left(u_{W}-u\right)^{1 / 2} \exp \left[(i \nu / \beta a)\left(u_{W}-u\right)\right]} \\
\quad \times \int_{v_{1}(u)}^{v_{*}(u)} \frac{\left[\Lambda_{B}(u, v)+\Lambda_{T}(u, v)\right] \cos \left\{(2 v / M \beta a)\left[\left(u_{W}-u\right)\left(v_{W}-v\right)\right]^{1 / 2}\right\}}{2\left(v_{W}-v\right)^{1 / 2} \exp \left[(i \nu / \beta a)\left(v_{W}-v\right)\right]} d v \\
\quad-\frac{U}{\pi M} \exp (i \nu t) \int_{u^{\prime}}^{u{ }^{W}} \frac{d u}{\left(u_{W}-u\right)^{1 / 2} \exp \left[(i \nu / \beta a)\left(u_{W}-u\right)\right]} \\
\quad \times \int_{v_{1}(u)}^{v_{W}} \frac{\Lambda_{T}(u, v) \cos \left\{(2 \nu / M \beta a)\left[\left(u_{W}-u\right)\left(v_{W}-v\right)\right]^{1 / 2}\right\}}{\left(v_{W}-v\right)^{1 / 2} \exp \left[(i \nu / \beta a)\left(v_{W}-v\right)\right]} d v .
\end{aligned}
$$

Eq. 35 may be restated in the following theorem:

Theorem 4. The velocity potential, in the mixed supersonic region on the top surface of a three-dimensional oscillating wing, may be computed by Eq. 35 or, in the $x, y$ coordinates,

$$
\begin{aligned}
& \Phi(x, y,+0, t)=-\frac{U}{\pi} \exp (i \nu t) \\
& \quad \times \iint_{W_{1}} \frac{\left[\Lambda_{B}(\xi, \eta)+\Lambda_{T}(\xi, \eta)\right] \cos \left\{\left(\nu / \beta^{2} a\right)\left[(x-\xi)^{2}-\beta^{2}(y-\eta)^{2}\right]^{1 / 2}\right\}}{2\left[(x-\xi)^{2}-\beta^{2}(y-\eta)^{2}\right]^{1 / 2} \exp \left[\left(i \nu U / \beta^{2} a^{2}\right)(x-\xi)\right]} d \eta d \xi \\
& \quad-\frac{U}{\pi} \exp (i \nu t) \iint_{S_{W:}} \frac{\Lambda_{T}(\xi, \eta) \cos \left\{\left(\nu / \beta^{2} a\right)\left[(x-\xi)^{2}-\beta^{2}(y-\eta)^{2}\right]^{1 / 2}\right\} d \eta d \xi}{\left[(x-\xi)^{2}-\beta^{2}(y-\eta)^{2}\right]^{1 / 2}} \frac{\exp \left[\left(i \nu U / \beta^{2} a^{2}\right)(x-\xi)\right]}{\exp }
\end{aligned}
$$


where $S_{W_{1}}$ is the area bounded by $0 \leq u \leq u^{\prime}$ and $v_{1}(u) \leq v \leq v_{2}(u)$ and $S_{W_{2}}$ is the area bounded by $u^{\prime} \leq u \leq u_{W}$ and $v_{1}(u) \leq v \leq v_{W}$.

The corresponding result for a point on the bottom surface of the wing may be obtained by interchanging $\Lambda_{T}$ and $\Lambda_{B}$. It may be noted that for a wing of zero thickness, $\Lambda_{B}(\xi, \eta)+\Lambda_{T}(\xi, \eta)=0$ so the integrals over $S_{W_{1}}$ vanish. The simple "equivalent area" theorem established by Evvard in Ref. 2 for steady flows is thus seen to be valid for oscillating flows.

Discussion. (A) In Ref. 4, the boundary value problem for the determination of the velocity potential in the purely supersonic region of a wing in unsteady motion at supersonic speed was treated by source-superposition method in a quite general manner. In fact, theorems 1 and 2 mentioned above are included in Garrick and Rubinow's results. On specializing to considerations of an oscillating wing, the derivation of Eq. 16 becomes very simple. The derivation of the same equation in Ref. 4 is more complicated.

(B) Since an arbitrary downwash function can usually be expanded as a Fourier series, a harmonically oscillatory motion may be considered as a basis for building up more general motion for a nonstationary wing. This paves the way to construct a proof for a theorem applicable to more general nonstationary wings. Evvard, in Ref. 1, treated

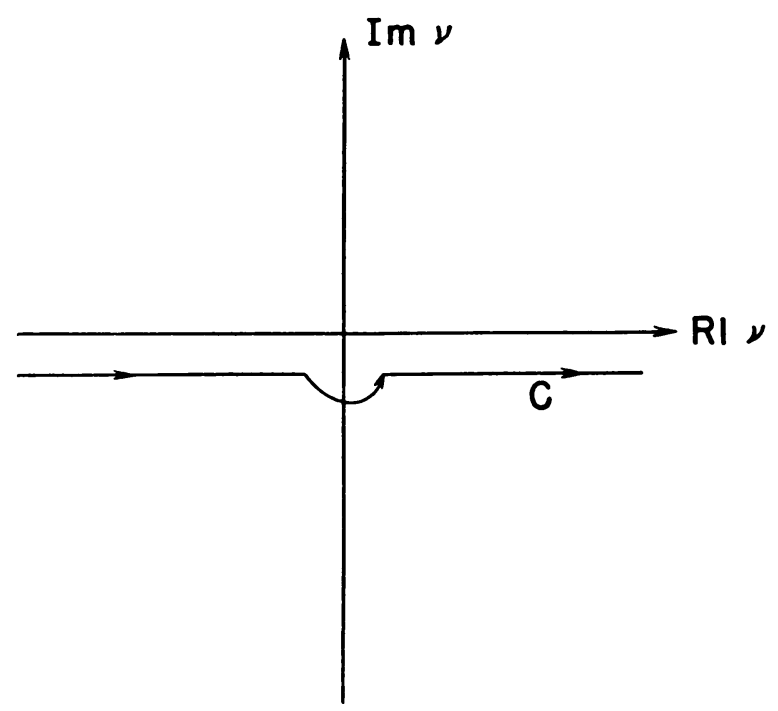

Fig. 5. Contour $C$ in $\nu$-plane.

the general mixed boundary value problem by the source superposition method. His results include the present theorems 3 and 4; however, his analysis was not carried through to the present point.

(C) A particular type of motion which is of both theoretical and practical interest and which demonstrates simply that theorems 3 and 4 apparently do not apply in the simple equivalent area form to all nonsteady motions, is the so-called "unit step" motion, in which a wing at rest starts abruptly at a certain instant and then maintains a steady motion. For composition of the velocity potential for a wing with motion of this nature, the "unit step" source will be useful. The "unit step" source can be derived from an oscillating source by a contour integration in the $\nu$ plane, 
$\phi_{2}(x, y, z, t)=\frac{1}{2 \pi i} \int_{C} \varphi_{1} \frac{d \nu}{\nu}=\frac{\beta}{2 \pi i r_{1}} \int_{C} \cos \left(\frac{\nu r_{1}}{\beta^{2} a}\right) \exp \left\{i \nu\left[t-\frac{U}{\beta^{2} a^{2}}(x-\xi)\right]\right\} \frac{d \nu}{\nu}$,

where $C$ is the contour shown in Fig. 5. By writing the cosine term in exponential form Eq. 36 can be shown to yield

$$
\phi_{2}(x, y, z, t)=\frac{\beta}{2 r_{1}}\left[H\left(t-\frac{U x}{\beta^{2} a^{2}}+\frac{r_{1}}{\beta^{2} a}\right)+H\left(t-\frac{U x}{\beta^{2} a^{2}}-\frac{r_{1}}{\beta^{2} a}\right)\right],
$$

where $H(\mu)$ is the "unit step" function having the properly that

$$
H(\mu)= \begin{cases}1 & \mu>0, \\ 0 & \mu<0 .\end{cases}
$$

Now, draw a sphere of radius (at) enclosed in the circular cone from the "unit step" source at $(\xi, \eta, \zeta)$, with the center of the sphere located at a distance $(U t)$ from $(\xi, \eta, \zeta)$

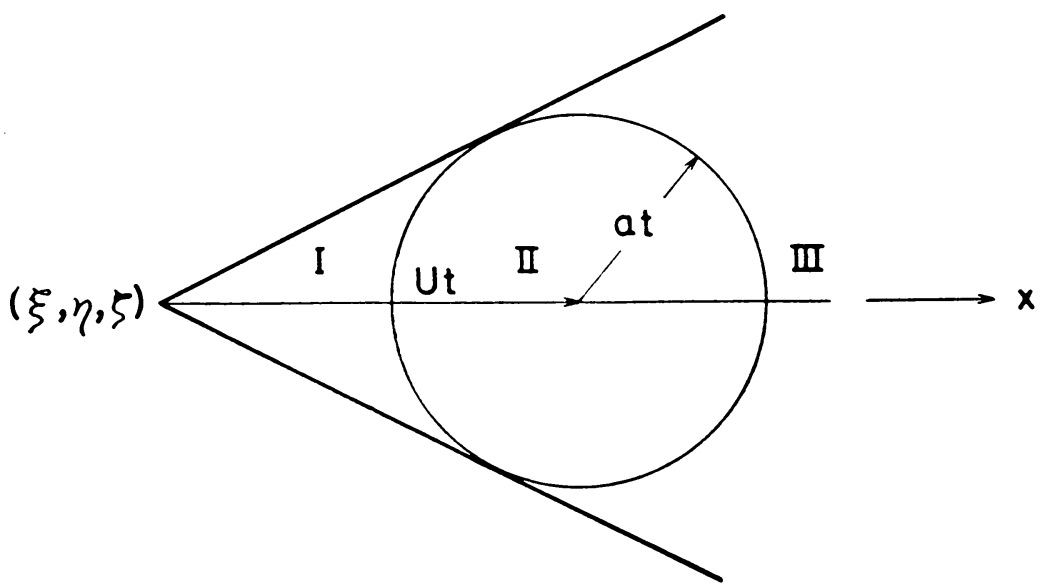

FIG. 6. Region of influence of the "unit step" source at $(\xi, \eta, \zeta)$ at instant $t$.

(Fig. 6). Then the region of influence of the source is divided into three regions (by Eq. 37),

(1) In region I, the influence is equivalent to that of a steady source.

(2) In region II, the influence is equivalent to that of a steady source of half strength.

(3) In region III, no influence of the source will be felt.

The region of dependence for a point $(x, y, z)$ will consist of three similar regions.

Consider the lift problem of a rectangular flat plate wing performing a "unit step" motion. Suppose that the velocity potential at a point $S$ in the mixed supersonic region near the wing tip is to be computed at an instant $t_{1}$, such that $a t_{1}<|y|$ (Fig. 7). According to the above argument, the condition at $S$ will depend on both regions $A$ and $B$ and the wing tip will have no influence. But in accordance with equivalent area form of Theorem 4 the domain of dependence at $S$ would exclude the shaded region in Fig. 7 in the computation of the velocity potential at $S$, at instant $t_{1}$. This provides an example 
of the fallacy of the equivalent area interpretation of Theorem 4 for arbitrary nonsteady motions.

Therefore, theorems 3 and 4 are not directly applicable to "unit step" wings. This fact is indicated (but not proved) by Eq. 36, because the operation of the contour inte-

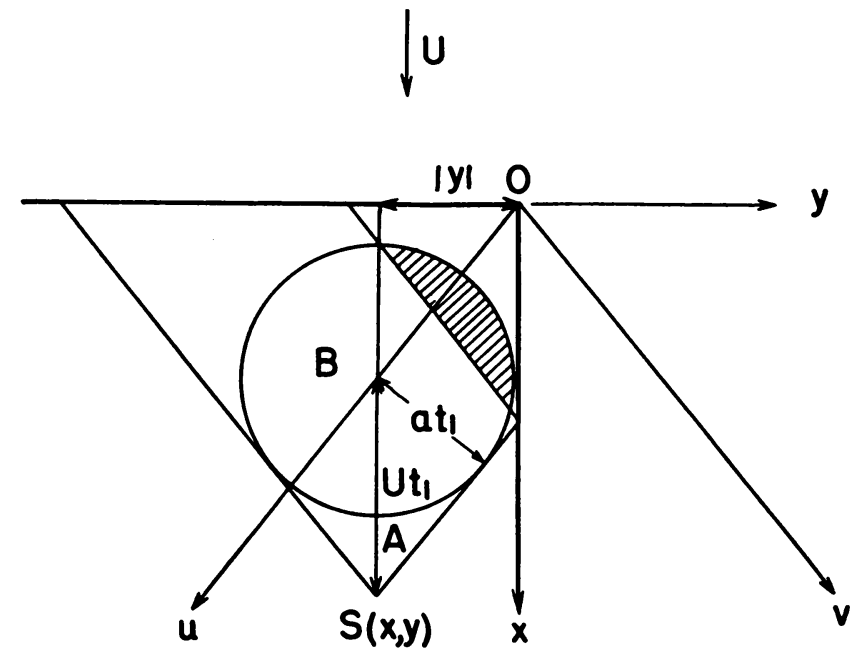

FIG. 7. The wing tip region of a rectangular flat plate performing "unit step" motion at instant $t_{4}$.

gration will carry the cosine function to infinity such that the argument of Eq. 25b breaks down in the proof of Theorem 3.

\section{REFERENCES}

1. Evvard, J. C., A linearized solution for time-dependent velocity potentials near three-dimensional wings at supersonic speeds, NACA TN 1699, Sept. 1948.

2. Evvard, J. C., Distribution of wave drag and lift in the vicinity of wing tips at supersonic speeds, NACA TN No. 1382, July 1947.

3. Evvard, J. C., and Turner, L. R., Theoretical lift distribution and upwash velocities for thin wings at supersonic speeds, NACA TN No. 1484, November 1947.

4. Garrick, I. E., and Rubinow, S., Theoretical study of air forces on an oscillating or steady thin wing in a supersonic main stream, NACA TN No. 1383, July 1947.

5. Stewart, H. J., The lift of a delta wing at supersonic speeds, Q. Appl. Math. 4, 246 (1946).

6. Miles, J. W., The aerodynamic forces on an oscillating airfoil at supersonic speeds, J. Aero. Sci. 14, 351 (1947).

7. Miles, J. W., Harmonic and transient motion of a swept wing in supersonic flow, J. Aero. Sci. 15, 343 (1948).

8. Whittaker, E. T., and Watson, G. N. A course of modern analysis, University Press, Cambridge, 1945. 